\title{
Isolation and Expression of Glucosinolate Synthesis Genes CYP83A1 and CYP83B1 in Pak Choi (Brassica rapa L. ssp. chinensis var. communis (N. Tsen \& S.H. Lee) Hanelt)
}

\author{
Biao Zhu ${ }^{1}$, Zhizhou Wang ${ }^{1}$, Jing Yang ${ }^{2}$, Zhujun Zhu ${ }^{1,2} *$ and Huasen Wang ${ }^{2}$
}

1 Department of Horticulture, Zhejiang University, Zijingang Campus, Yuhangtang Road 866, Hangzhou 310058, China; E-Mails: billzhu@zju.edu.cn (B.Z.); twzz@163.com (Z.W.)

2 Department of Horticulture, School of Agricultural and Food Science, Zhejiang A \& F University, Huan Cheng Bei Lu 88, Lin'an, Hangzhou 311300, China; E-Mails: yangjing@zafu.edu.cn (J.Y.); whsych66@163.com (H.W.)

* Author to whom correspondence should be addressed; E-Mail: zhjzhu@zju.edu.cn or zhuzj@zafu.edu.cn; Tel.: +86-571-6374-3001; Fax: +86-571-6374-1276.

Received: 6 February 2012; in revised form: 22 April 2012 / Accepted: 27 April 2012 / Published: 15 May 2012

\begin{abstract}
C Y P 83 A 1$ and $C Y P 83 B 1$ are two key synthesis genes in the glucosinolate biosynthesis pathway. CYP83A1 mainly metabolizes the aliphatic oximes to form aliphatic glucosinolate and $C Y P 83 B 1$ mostly catalyzes aromatic oximes to synthesis corresponding substrates for aromatic and indolic glucosinolates. In this study, two CYP83A1 genes named BcCYP83A1-1 (JQ289997), BcCYP83A1-2 (JQ289996) respectively and one CYP83B1 (BcCYP83B1, HM347235) gene were cloned from the leaves of pak choi (Brassica rapa L. ssp. chinensis var. communis (N. Tsen \& S.H. Lee) Hanelt) "Hangzhou You Dong Er" cultivar. Their ORFs were 1506, 1509 and 1500 bp in length, encoding 501, 502 and 499 amino acids, respectively. The predicted amino acid sequences of CYP83A1-1, CYP83A1-2 and CYP83B1 shared high sequence identity of $87.65,86.48$ and $95.59 \%$ to the corresponding ones in Arabidopsis, and 98.80, 98.61 and $98.80 \%$ to the corresponding ones in Brassica pekinensis (Chinese cabbage), respectively. Quantitative real-time PCR analysis indicated that both $C Y P 83 A 1$ and $C Y P 83 B 1$ expressed in roots, leaves and petioles of pak choi, while the transcript abundances of $C Y P 83 A 1$ were higher in leaves than in petioles and roots, whereas $C Y P 83 B 1$ showed higher abundances in roots. The expression levels of glucosinolate biosynthetic genes were consistent with the glucosinolate profile accumulation in shoots of seven cultivars and three organs. The isolation and
\end{abstract}


characterization of the glucosinolate synthesis genes in pak choi would promote the way for further development of agronomic traits via genetic engineering.

Keywords: pak choi; glucosinolate; phylogenetic analysis; quantitative real-time PCR

\section{Introduction}

Pak choi (Brassica rapa L. ssp. chinensis var. communis (N. Tsen \& S.H. Lee) Hanelt) is a very important vegetable crop in China and Northeast Asia because of its high yield and nutritional value [1]. It contains a number of bioactive components such as folates, vitamin $\mathrm{C}$, carotenoids, polyphenols and glucosinolates which have anti-carcinogenic effects [2-4]. Glucosinolates are a group of plant secondary metabolites derived from different kinds of amino acids and can be hydrolyzed by myrosinase to produce a variety of compounds which can have beneficial effects on human health including anti-carcinogenic, cholesterol-reducing and other pharmacological effects [5]. The glucosinolates-myrosinase-system is also considered to be an efficient defense system for plant against herbivorous generalists and microorganisms [6-8].

To date, more than 132 individual glucosinolates have been detected, and they are grouped into aliphatic, aromatic and indole glucosinolates depending on the structure of their side-chain [9]. In recent years, significant advances have been made in our understanding of the biosynthetic pathway of glucosinolates. The formation of glucosinolates can be divided into three separate phases including the chain elongation, formation of the core structure and secondary modifications of side chain and glucose moiety of the parent glucosinolates [10]. Since the completion of the Arabidopsis genome sequencing, a significant progress on glucosinolate research has been made especially in the identification of the genes and regulators for glucosinolate biosynthesis. MAM (Methylthioalkylmalate synthase) genes are responsible for the chain-elongation. CYP79 enzymes catalyze the first committed step of glucosinolates core structure formation as the conversion of amino acids to aldoximes. CYP83 enzymes catalyze the key step for the core structure formation as the conversion of aromatic oximes, and the conversion of thiohydroximic acids to glucosinolates [11-18].

Of the two oxime-metabolizing enzymes CYP83A1 and CYP83B1, major progress has been made by using Arabidopsis as model plant. Aliphatic oximes derived from chain-elongated homologs of methionine are efficiently metabolized by CYP83A1 enzyme, whereas CYP83B1 enzyme metabolizes these substrates with very low efficiency. Aromatic oximes derived from phenylalanine, tryptophan, and tyrosine are metabolized by both enzymes, although CYP83B1 has higher affinity for these substrates than CYP83A1 [19,20]. However, most of these researches are carried out in the model plant Arabidopsis. As glucosinolates have been proved to have many beneficial effects on human health and Arabidopsis is not edible, it is necessary to focus on glucosinolates-containing crops which are important for human consumption. In the present study, two glucosinolates biosynthesis genes $B c C Y P 83 A 1$ and $B c C Y P 83 B 1$ were isolated from pak choi and characterized and their expression pattern in different organs and cultivars were also characterized. 


\section{Results and Discussion}

\subsection{Identification and Sequence Analysis of CYP83A1 and CYP83B1}

Two aliphatic oxime-metabolizing enzymes namely $B c C Y P 83 A 1-1, B c C Y P 83 A 1-2$, and one aromatic oxime-metabolizing enzyme $B c C Y P 83 B 1$ were isolated from the leaves of pak choi "Hangzhou You Dong Er" cultivar. The ORF of the genes were 1506, 1509 and 1500 bp in length, encoding 501, 502 and 499 amino acids, respectively (Figures 1 and 2). A BLAST search revealed that the primary structure of CYP83A1-1, CYP83A1-2 and CYP83B1 shared high sequence identity of 86.81, 87.43 and 90.13\% to the corresponding ones in Arabidopsis (NM117451, NM119299), respectively, and shared high sequence identity of $99.14,99.07$ and $99.47 \%$ to the corresponding ones in Brassica pekinensis (Brassica rapa L. ssp. pekinensis var. communis, Chinese cabbage, FJ376049, FJ376050, FJ376051) respectively. The amino acid sequence deduced from CYP83A1-1 and CYP83A1-2 showed 87.65 and $86.48 \%$ identity to that of the Arabidopsis CYP83A1, and 98.80 and $98.61 \%$ identity to Brassica pekinensis CYP83A1-1 and CYP83A1-2, respectively. The deduced amino acid sequence of CYP83B1 showed $95.59 \%$ and $98.80 \%$ identity to that of the Arabidopsis and Brassica pekinensis, respectively. The sequence similarity between CYP83A1-1 and CYP83A1-2 were $85.88 \%$ in this study, identical to that of $85.29 \%$ in Brassica pekinensis.

Figure 1. Multiple alignment of deduced amino acid sequences of $C Y P 83 A 1$. Black shaded and other shaded boxes show identical and similar amino acids, respectively. GenBank accession number of the CYP83A1s: Arabidopsis thaliana (NM117451), Brassica pekinensis (FJ376049, FJ376050) and Brassica chinensis (JQ289997, JQ289996).

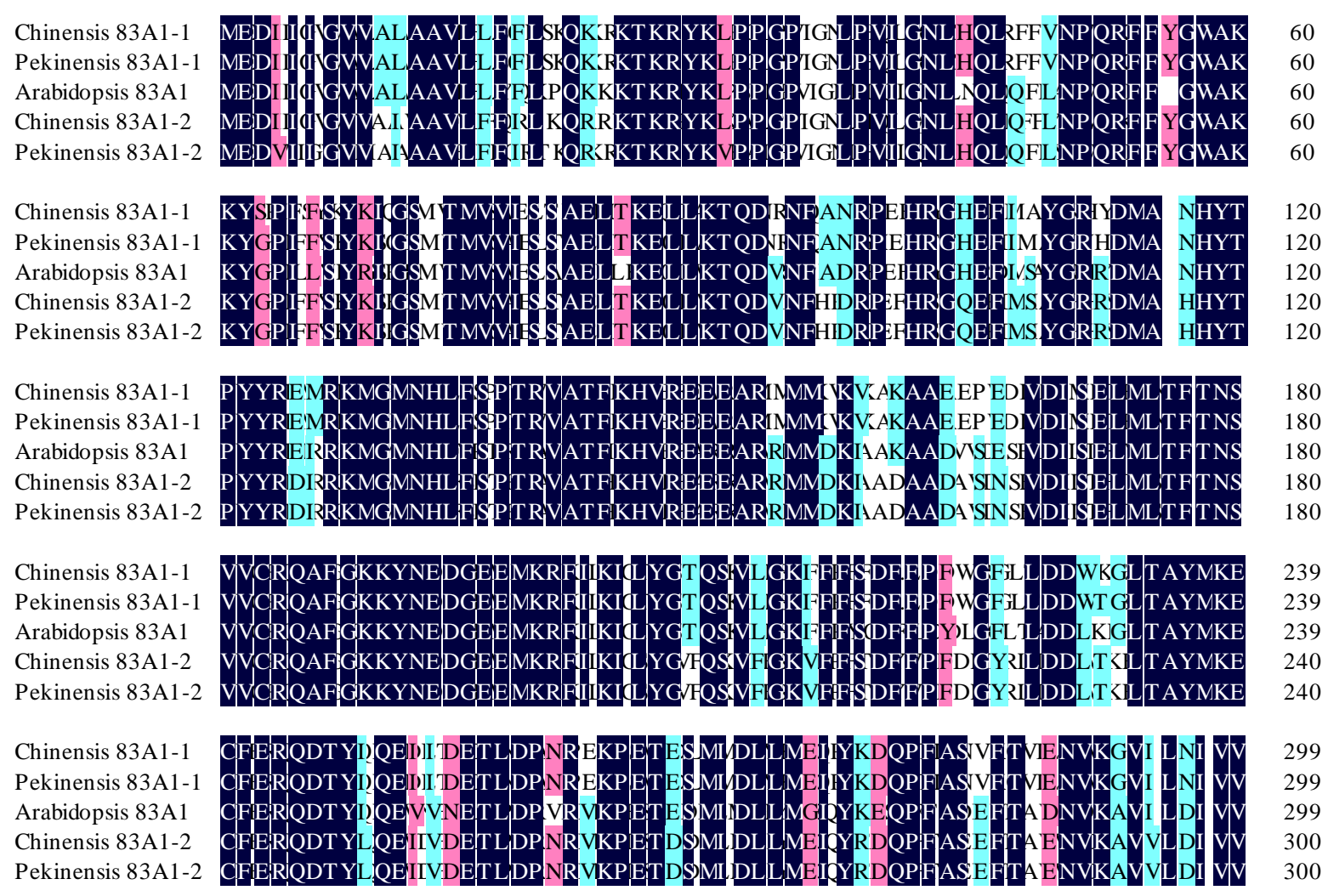


Figure 1. Cont.

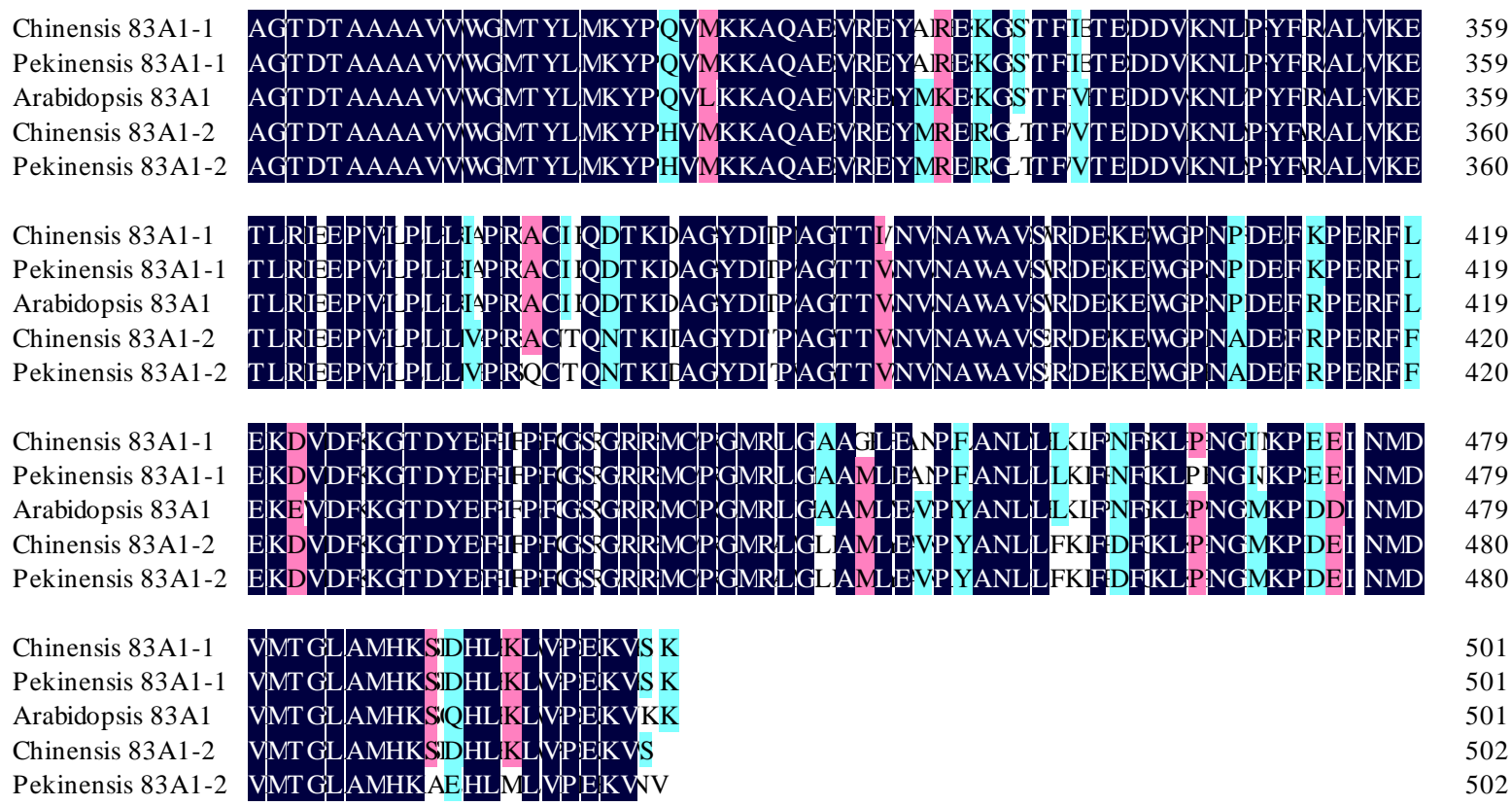

Figure 2. Multiple alignment of deduced amino acid sequences of CYP83B1. Black shaded and other shaded boxes show identical and similar amino acids, respectively. GenBank accession number of the CYP83B1s: Arabidopsis thaliana (NM119299), Brassica pekinensis (FJ376051) and Brassica chinensis (HM347235).

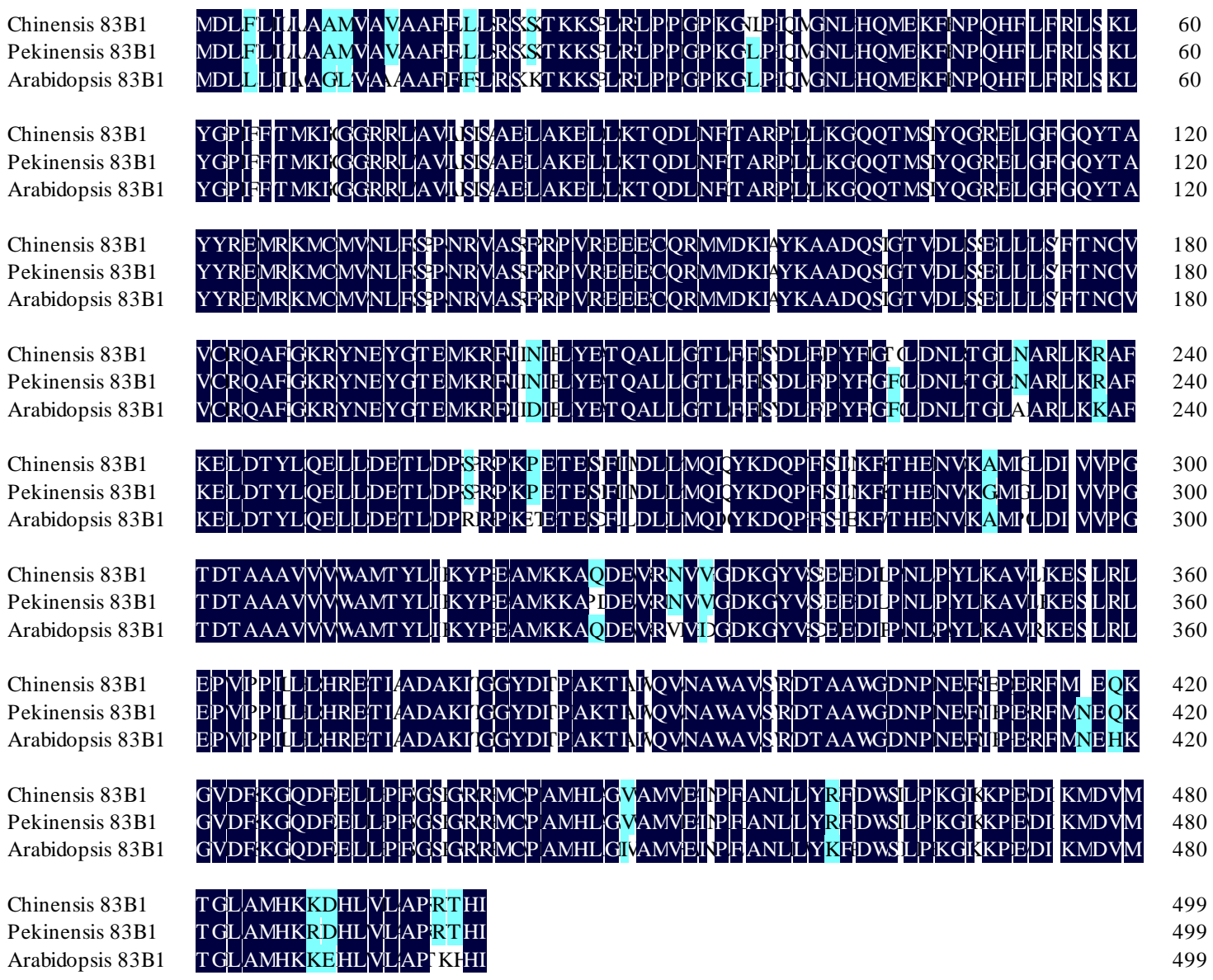




\subsection{Phylogenetic Analysis of CYP83A1 and CYP83B1}

We compared the isolated sequences with the corresponding ones in Arabidopsis thaliana (NM117451, NM119299) and Brassica pekinensis (FJ376049, FJ376050, FJ376051) by multiple alignments, and reconstructed the phylogenetic tree by ClustalW analysis program. The results showed that the homologs of CYP83A1 and CYP83B1 from the Brassicaceae family are highly conserved, with low variation in DNA sequence. As shown in the phylogenetic tree, the glucosinolate synthesis genes of Brassica chinensis and Brassica pekinensis clustered first with each other and then with the genes of Arabidopsis (Figure 3), which demonstrated the highly congruent relationship among different sources of glucosinolate synthesis genes.

Figure 3. Phylogenetic tree of the cDNA sequences of the plant glycosyltransferase superfamily. GenBank accession numbers are: Arabidopsis thaliana CYP83A1 (NM117451), Arabidopsis thaliana CYP83B1 (NM119299), Brassica pekinensis CYP83A1-1 and CYP83A1-2 (FJ376049, FJ376050), Brassica pekinensis CYP83B1 (FJ376051), Brassica chinensis CYP83A1-1 and CYP83A1-2 (JQ289997, JQ289996), Brassica chinensis CYP83B1 (HM347235). The horizontal scale shows the number of differences per 100 residues derived from the ClustalW alignment.

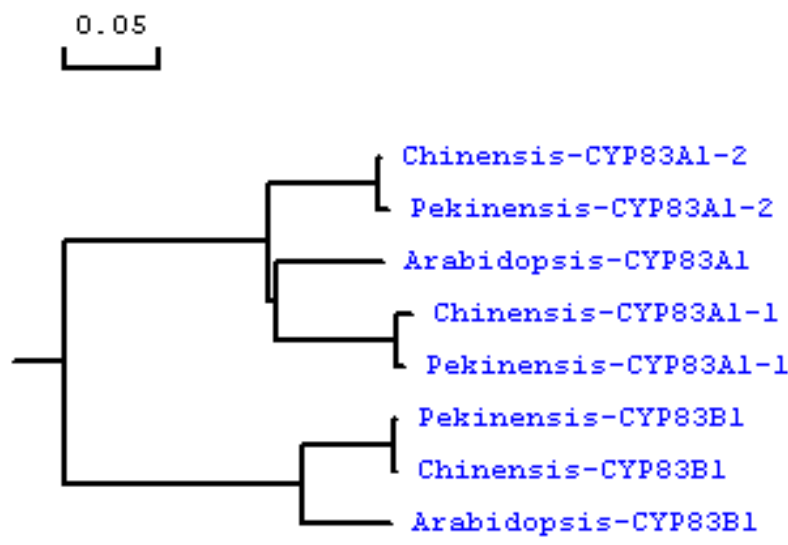

\subsection{Expression of CYP83A1 and CYP83B1 in Different Cultivars and Various Organs}

Quantitative real-time PCR (qRT-PCR) was employed to confirm the expression patterns of the two genes in seven cultivars, as well as in different organs. Actin was used as an internal reference control for total RNA input. In our analysis, the gene-specific primers used for amplification of $C Y P 83 A 1$ recognized and amplified both CYP83A1-1 and CYP83A1-2 genes. Both CYP83A1 and CYP83B1 were detected in the leaves of all the seven cultivars and had the same expression pattern except for cultivar "ZYYDE". The expression patterns of the two genes in leaves of seven cultivars are illustrated in Figure 4. CYP83A1 was presented in higher abundance compared with CYP83B1 in most of the cultivars, whereas the $C Y P 83 A 1$ and $C Y P 83 B 1$ transcripts in "ZYYDE" cultivar were nearly same. 
Figure 4. Relative expression analysis of $C Y P 83 A 1$ and $C Y P 83 B 1$ in leaves of seven cultivars of pak choi. Error bars represent the standard deviation (SD) from three biological repeats.

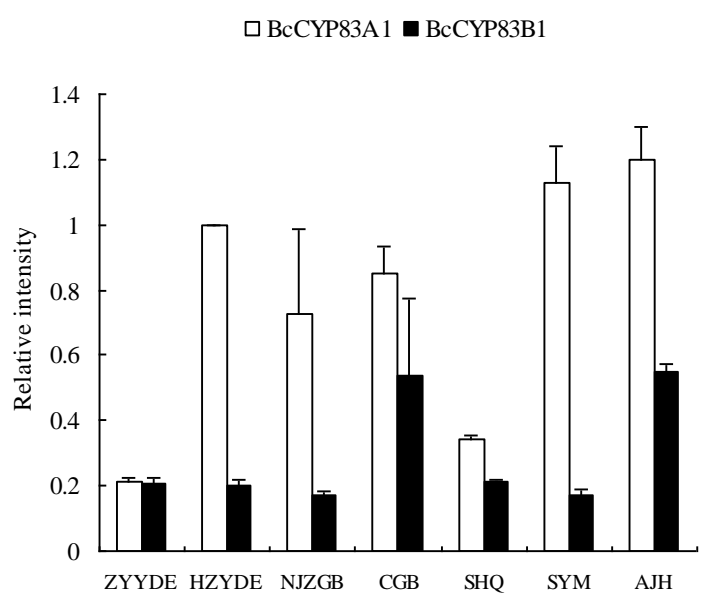

Both the aliphatic oxime-metabolizing enzyme and aromatic oxime-metabolizing enzyme were identified in the three organs (roots, leaves and petioles) of pak choi (cv. HZYDE). Though CYP83A1 and $C Y P 83 B 1$ exist in all those organs, they displayed spatial expression patterns (Figure 5). The expression level of CYP83A1 was higher in leaves than in roots or petioles, whereas the CYP83B1 showed higher mRNA abundance in roots compared with another two.

Figure 5. Relative expression analysis of $C Y P 83 A 1$ and $C Y P 83 B 1$ in leaf, petiole and root of pak choi cv. HZYDE. Error bars represent the standard deviation (SD) from three biological repeats.

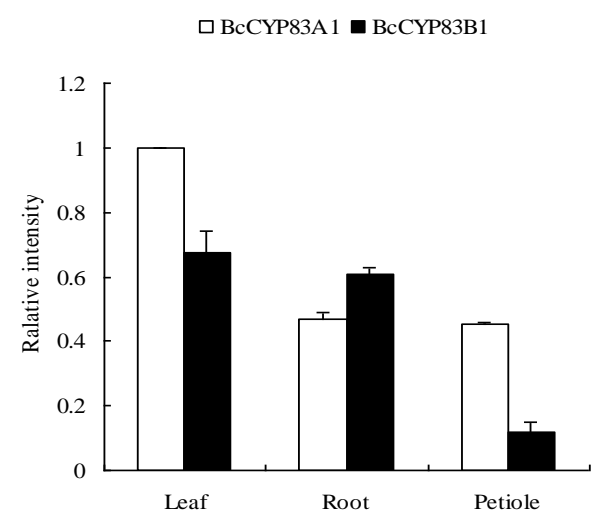

\subsection{Glucosinolate Profile Concentrations in Different Cultivars and Various Organs}

The concentrations of aliphatic, indole and aromatic glucosinolates in different pak choi cultivars and in different organs of "Hangzhou You Dong Er" cultivar were analyzed. As shown in Table 1, the aliphatic glucosinolate concentrations in leaves of most of the cultivars were higher than indole and aromatic glucosinolates. The glucosinolate profiles in different organs varied differently. As leaf and petiole had particularly higher aliphatic glucosinolates, however, root had much higher indole and aromatic glucosinolates. 
Table 1. Aliphatic, indole and aromatic glucosinolates content $(\mu \mathrm{mol})$ in shoots of different cultivars and in different organs of "Hangzhou You Dong Er" cultivar. Data are the means of three biological repeats \pm standard deviation.

\begin{tabular}{|c|c|c|c|c|c|c|c|c|c|c|}
\hline & ZYYDE & HZYDE & NJZGB & CGB & SHQ & SYM & AJH & Leaf & Root & Petiole \\
\hline Aliphatic GS & $0.43 \pm 0.07$ & $0.94 \pm 0.07$ & $0.49 \pm 0.07$ & $0.54 \pm 0.08$ & $0.98 \pm 0.04$ & $2.90 \pm 0.34$ & $0.87 \pm 0.10$ & $1.89 \pm 0.29$ & $0.12 \pm 0.02$ & $0.48 \pm 0.05$ \\
\hline Indole GS & $0.68 \pm 0.03$ & $0.76 \pm 0.03$ & $0.30 \pm 0.01$ & $0.45 \pm 0.02$ & $0.52 \pm 0.02$ & $0.59 \pm 0.03$ & $0.75 \pm 0.10$ & $0.25 \pm 0.06$ & $0.94 \pm 0.10$ & $0.24 \pm 0.04$ \\
\hline Aromatic GS & $0.38 \pm 0.02$ & $0.51 \pm 0.02$ & $0.31 \pm 0.03$ & $0.59 \pm 0.07$ & $0.63 \pm 0.04$ & $0.46 \pm 0.07$ & $0.61 \pm 0.05$ & $0.28 \pm 0.06$ & $0.78 \pm 0.07$ & $0.25 \pm 0.03$ \\
\hline Total GS & $1.49 \pm 0.07$ & $2.21 \pm 0.09$ & $1.10 \pm 0.09$ & $1.58 \pm 0.11$ & $2.13 \pm 0.05$ & $3.95 \pm 0.41$ & $2.23 \pm 0.04$ & $2.43 \pm 0.24$ & $1.84 \pm 0.17$ & $0.97 \pm 0.05$ \\
\hline
\end{tabular}

\subsection{Discussion}

In this study, we isolated two CYP83A1 genes and one $C Y P 83 B 1$ gene from pak choi "Hangzhou You Dong Er" cultivar. Amino acid sequence analysis and sequence alignment with their homologs from Brassica pekinensis and Arabidopsis showed the two key glucosinolate synthetic genes CYP83A1 and CYP83B1 within Brassicaceae family are highly conserved. As shown in Figures 1 and 2, the genes isolated from pak choi have both high DNA and amino acid sequence conservation with their Brassica pekinensis counterparts. The identity of DNA sequence and deduced amino acid sequence of CYP83B1 between the two species was $99.47 \%$ and $98.80 \%$. The two copies of CYP83A1 namely $B c C Y P 83 A 1-1$ and $B c C Y P 83 A 1-2$ from pak choi were also highly similar to the corresponding ones of Brassica pekinensis, whereas there was only one copy of CYP83A1 in Arabidopsis. This is consistent with the previous finding by Zang et al. that identities within Brassica rapa were usually higher than those with Arabidopsis [21]. They isolated and analysed the glucosinolate synthesis genes in Brassica pekinensis by genome-wide identification [21]. The result was also accordant to the notion that Brassica species evolved and were triplicated after divergence from Arabidopsis lineage [22-24]. The observed glucosinolate synthesis sequence similarities among the Brassicas (mostly >90\%) were higher than those between Brassica and Arabidopsis (mostly 80-90\%) in this study also supported the presumptive evolution order.

Consistent with the results of previous studies in Arabidopsis, both the CYP83B1 and CYP83AI transcripts were expressed in all tested organs (roots, leaves and petioles) of pak choi [25,26]. Mizutani reported that CYP83Al expression levels in Arabidopsis was highest in leaves and significantly higher in roots and stems than in flowers and siliques, while CYP83B1 was the most highly expressed in roots [25]. In our study, the expression level of $C Y P 83 A 1$ was also highest in leaves, and the CYP83B1 expressed higher in leaves and roots than in petioles. These results were consistent with the previous finding that CYP83A1 expressed highest in the leaves and CYP83B1 were preferentially expressed in the roots $[25,26]$. The gene expression pattern in the organs was consistent with the glucosinolate profile accumulations as aliphatic glucosinolates were higher in leaves and petioles while indole and aromatic glucosinolates were higher in roots. The results were similar to Brown's finding that aliphatic glucosinolates are typically higher in the leaves compared to the roots while indole glucosinolates are abundant in the roots [27]. When compared within an appointed organ, we found that the expression patterns of the two genes were different. CYP83A1 transcripts were almost two-folds of CYP83B1 transcripts in leaves and petioles, in contrast, the expression levels of the CYP83A1 were relatively lower than CYP83B1 in roots. Our finding was also accordant to the conclusion that the CYP83A1 
catalyzed aliphatic glucosinolates biosynthesis and accumulation in leaves and the CYP83B1 catalyzed aromatic and indole glucosinolates mostly found in roots [15].

The expression patterns of the two genes in leaves of seven cultivars in our study were similar except "ZYYDE" cultivar and the gene expression levels were consistent with the glucosinolate profile accumulation. The expression levels of $C Y P 83 A 1$ in leaves of six of seven cultivars were higher than $C Y P 83 B 1$ and the aliphatic glucosinolates of the six CYP83A1 higher expression cultivars were higher than another two glucosinolate profiles, whereas $C Y P 83 A 1$ and $C Y P 83 B 1$ expressed nearly identical in "ZYYDE" cultivar. While focused on the specific gene expression and glucosinolate accumulation in individual cultivar, "SYM" cultivar had high CYP83A1/CYP83B1 transcript level ratio and high aliphatic glucosinolates/indole glucosinolates ratio. And "CGB" cultivar had the higher CYP83B1 transcript level and relative higher aromatic glucosinolates accumulation.

\section{Experimental Section}

\subsection{Plant Materials}

The seeds of seven pak choi cultivars (Brassica rapa L. ssp. chinensis var. communis (N. Tsen \& S.H. Lee) Hanelt) with different characters were sown in plastic pot with vermiculite in a growth chamber with $26 / 22{ }^{\circ} \mathrm{C}$ ( $12 \mathrm{~h}$ light/12 h dark) for 2 weeks. The seven commonly plant and consumed pak choi cultivars in southern China used are "Hangzhou You Dong Er" (HZYDE), "Zhou Ye You Dong Er" (ZYYDE), "Si Yue Man" (SYM), "Shanghai Qing” (SHQ), "Nanjing Zhong Gan Bai" (NJZGB), "Chang Geng Bai" (CGB) and "Ai Jiao Huang" (AJH). The young leaves from two-week old plants of seven pak choi cultivars were collected as experimental materials for quantitative real-time PCR (qRT-PCR) analysis. And three organs including roots, petioles and leaves of "Hangzhou You Dong Er" cultivar were collected for the expression analysis.

\subsection{Isolation of Total RNA and Synthesis of Full-Length cDNA Sequence}

Total RNA was extracted from samples using TRIzol reagent RNAisoTM Plus (Takara, D9108A) following the manufacturer's protocols. Approximately $0.1 \mathrm{~g}$ of tissue was extracted in $1 \mathrm{~mL}$ of extraction buffer. After melted in RNA-free water, RNA was quantified by UV spectroscopy and its integrity was visually assessed on ethidium bromide stained agarose gels. The cDNA was synthesized from 500 ng of DNA-free RNA with a PrimeScript RT reagent Kit (Takara, DRR037A) following the manufacturer's protocol.

Degenerate primers allowing amplification were designed based on sequences corresponding to highly conserved peptide regions of CYP83A1 and CYP83B1 of Arabidopsis and Brassica pekinensis. The cDNAs were amplified by PCR using the degenerate primers, then cloned into the pMD18-T Easy vector (Takara, Code D101A) and sequenced (Invitrogen). Rapid amplification of cDNA ends (RACE) was performed to obtain the $3^{\prime}$ and 5' ends of the genes with the SMART RACE cDNA Amplification Kit (Clontech) according to the manufacturer's instructions. Sequence analysis of open reading fragment (ORF) and encoded amino acid sequence of genes were deduced by BioXM 2.6 (Msight, Guangzhou, China, 2007). Physicochemical properties of the deduced protein were predicted by Protparam [28]. 


\subsection{Phylogenetic Analysis}

The nucleotide and deduced amino acid sequences were used for multiple alignments and phylogenetic tree analysis. The tree was obtained by ClustalW analysis program [29].

\subsection{Quantitative Real-Time RT-PCR}

The oligonucleotide primers for quantitative real-time PCR (qRT-PCR) analysis were designed with primer3 (version 0.4.0) based on the gene sequences (JQ289997, JQ289996, HM347235 and EX087730) [30]. For CYP83A1, we designed a specific primer which can amplify both CYP83A1-1 and CYP83A1-2 for qRT-PCR according to the highly conserved peptide regions compared with Brassica chinensis, Brassica pekinensis and Arabidopsis. The qRT-PCR primers for CYP83B1 and actin gene were designed the same way. The size of all qPCR products was limited to 100-300 nucleotides. PCR product size was analyzed on $1 \%$ agarose gels stained with ethidium bromide and the specificity of qRT-PCR products was then confirmed by sequencing. The qPCR primers of CYP83A1, CYP83B1 and Actin were as follows respectively:

CYP83A1

(F) 5'-TCCTCTCCTTATCCCTCGTGCTTGC-3'

(R) 5'-CCAAGACGCATTCCAGGGCACATTC-3'

CYP83B1

(F) 5'-AGACTCTTGACCCTAGCCGTCCTA-3'

(R) 5'-CCTTTGTCACCGACCACATTCCT-3'

Actin

(F) 5'-CGCTTAACCCGAAAGCTAAC-3'

(R) 5'-TACGCCCACTAGCGTAAAGA-3'

For gene expression analysis, first-stranded cDNAs from the leaves of the seven cultivars, and first-stranded cDNAs from the leaves, the petioles and the roots of "HZYDE" cultivar were used as templates. RT-PCR was performed on Applied Biosystems 7300 Real-Time PCR system with the SYBR Green qPCR kit (Takara, DRR081A). Ten-fold diluted cDNA was used as the template in real-time PCR. $25 \mu \mathrm{L}$ total reaction volume comprised $12.5 \mu \mathrm{L} 2 \times$ SYBR Green PCR Master Mix (Takara), $1 \mu \mathrm{L}$ of each primer $(10 \mu \mathrm{M}), 2 \mu \mathrm{L}$ of cDNA, and $8.5 \mu \mathrm{L}$ of PCR-grade water. The PCR conditions were as the follows: initiated by $30 \mathrm{~s}$ at $95{ }^{\circ} \mathrm{C}$, then followed by 40 cycles of $95{ }^{\circ} \mathrm{C}$ for $5 \mathrm{~s}$, $60{ }^{\circ} \mathrm{C}$ for $31 \mathrm{~s}$, and completed with a melting curve analysis program. No-template controls and melting curve analyses were included in every reaction. Brassica actin (EX087730) was used as the housekeeping gene. A melting curve of denatured double-stranded cDNA was established to test the purity of the products, and the products were re-sequenced to confirm the specificity. Triplicate quantitative PCR experiments were performed for each sample, and the expression values obtained were normalized against actin. Analysis of the relative gene expression data was done using the $2^{-\Delta \Delta \mathrm{Ct}}$ method $[31,32]$. 


\subsection{Glucosinolate Analysis}

The glucosinolate extraction and analysis procedure was performed according to the method of Krumbein with slight modification [33]. Briefly, $0.25 \mathrm{~g}$ sample powder were boiled with $10 \mathrm{~mL} 70 \%$ methanol to inactive myrosinase, then the supernatants were loaded onto a $1 \mathrm{~mL}$ mini-column (JT Baker, Phillipsburg, NJ, USA) containing $500 \mu \mathrm{L}$ activated DEAE Sephadex ${ }^{\mathrm{TM}}$ A25 (Amersham Biosciences, Uppsala, Sweden) to de-sulphate overnight with aryl sulfatase (Sigma-Aldrich Co., St. Louis, MO, USA). The resultant desulpho (ds)-glucosinolates were eluted with ultra pure water and stored at $-20{ }^{\circ} \mathrm{C}$ prior to separation by high performance liquid chromatography (HPLC).

For HPLC, $20 \mu \mathrm{L}$ samples were analyzed in an Agilent 1200 HPLC system (G1311A quaternary pump, G1322A vacuum solvent delivery degasser, G1316A thermostatted column compartment, Agilent Technologies, Inc., Palo Alto, CA, USA) consisting of a G1329A auto injector, a prontosil ODS2 column $(250 \times 4 \mu \mathrm{m}, 5 \mu \mathrm{m}$, Bischoff, Leonberg, Germany) and a G1315B diode array detector (DAD) set at $229 \mathrm{~nm}$. The mobile phase was ultra pure water (A) and acetonitrile (Tedia, Fairfield, $\mathrm{OH}$, USA) (B) in a linear gradient from $0 \%$ to $20 \%$ B in $32 \mathrm{~min}$, then constant $20 \%$ B for 6 min, following by $100 \% \mathrm{~B}$ and $0 \% \mathrm{~B}$ prior to the injection of the next sample. The flow rate was $1.3 \mathrm{~mL} \cdot \mathrm{min}^{-1}$.

\section{Conclusions}

In the present study, we isolated and analyzed the glucosinolate biosynthetic genes in pak choi. This is the first report about glucosinolate genes in pak choi although they have been studied in Arabidopsis. The gene expression levels and glucosinolate profile accumulation were also compared. The isolation and further characterization of the genes responsible for the synthesis and regulation of glucosinolates in pak choi are important for the development of vegetables that are beneficial to health.

\section{Acknowledgments}

This research was financially supported by NSFC (30871718), ZJNSF (R3080360, Y3110340) and the fund for Zhejiang Higher School Innovative Research Team (T200916).

\section{References}

1. Tay, D.C.S.; Toxopeus, H. Brassica rapa L. cv. Group Pak Choi. In Plant Resources of South-East Asia, 8, Vegetables; Siemonsma, J.S., Piluek, K., Eds.; Pudoc Scientific Publishers: Wageningen, The Netherlands, 1993; pp. 130-134.

2. He, H.J.; Fingerling, G.; Schnitzler, W.H. Seasonal variations of the glucosinolate amounts in Brassica campestris L. spp. chinensis. J. Appl. Bot. 2000, 74, 198-202.

3. Lewis, L.; Fenwick, G.R. Glucosinolate content of brassica vegetables: Analysis of twenty-four cultivars of calabrese (green sprouting broccoli, Brassica oleracea L. var. botrytis subvar. cymosa Lam.). Food Chem. 1987, 25, 259-268.

4. Kurilich, A.C.; Tsau, G.J.; Brown, A.; Howard, L.; Klein, B.P.; Jeffery, E.H.; Kushad, M.; Wallig, M.A.; Juvik, J.A. Carotene, tocopherol and ascorbate contents in subspecies of Brassica oleracea. J. Agric. Food Chem. 1999, 47, 1576-1581. 
5. Mithen, R.F.; Dekker, M.; Verkerk, R.; Rabot, S.; Johnson, I.T. The nutritional significance, biosynthesis and bioavailability of glucosinolates in human foods. J. Sci. Food Agric. 2000, 80, 967-984.

6. Manici, L.M.; Lazzeri, L.; Palmieri, S. In vitro fungitoxicactivity of some glucosinolates and their enzyme-derived products towards plant pathogenic fungi. J. Agric. Food Chem. 1997, 45, 2768-2773.

7. Wittstock, U.; Gershenzon, J. Constitutive plant toxins and their role in defense against herbivores and pathogens. Curr. Opin. Plant Biol. 2002, 5, 300-307.

8. Wittstock, U.; Kliebenstein, D.J.; Lambrix, V.; Reichelt, M.; Gershenzon, J. Glucosinolate Hydrolysis and Its Impact on Generalist and Specialist Insect Herbivores. In Integrative Phytochemistry: From Ethnobotany to Molecular Ecology; Romeo, J.T., Ed.; Pergamon: Amsterdam, The Netherlands, 2003; pp. 101-125.

9. Agerbirk, N.; Olsen, C.E. Glucosinolate structures in evolution. Phytochemistry 2012, 77, 16-45.

10. Wittstock, U.; Halkier, B.A. Glucosinolate research in the Arabidopsis era. Trends Plant Sci. 2002, 7, 263-270.

11. Bak, S.; Feyereisen, R. The involvement of two P450 enzymes, CYP83B1 and CYP83A1, in auxin homeostasis and glucosinolate biosynthesis. Plant Physiol. 2001, 127, 108-118.

12. Barlier, I.; Kowalczyk, M.; Marchant, A.; Ljung, K.; Bhalerao, R.; Bennett, M.; Sandberg, G.; Bellini, C. The SUR2 gene of Arabidopsis thaliana encodes the cytochrome P450 CYP83B1, a modulator of auxin homeostasis. Proc. Natl. Acad. Sci. USA 2000, 97, 14819-14824.

13. Delarue, M.; Prinsen, E.; van Onckelen, H.; Caboche, M.; Bellini, C. Sur2 mutations of Arabidopsis thaliana define a new locus involved in the control of auxin homeostasis. Plant J. 1998, 14, 603-611.

14. Grubb, C.D.; Abel, S. Glucosinolate metabolism and its control. Trends Plant Sci. 2006, 11, 89-100.

15. Halkier, B.A.; Gershenzon, J. Biology and biochemistry of glucosinolates. Ann. Rev. Plant Biol. 2006, 57, 303-333.

16. Hansen, C.H.; Du, L.C.; Naur, P.; Olsen, C.E.; Axelsen, K.B.; Hick, A.J.; Pickett, J.A.; Halkier, B.A. CYP83B1 is the oxime-metabolizing enzyme in the glucosinolate pathway in Arabidopsis. J. Biol. Chem. 2001, 276, 24790-24796.

17. Piotrowski, M.; Schemenewitz, A.; Lopukhinat, A.; Mueller, A.; Janowitz, T.; Weiler, E.W.; Oecking, C. Desulfoglucosinolate sulfotransferases from Arabidopsis thaliana catalyze the final step in the biosynthesis of the glucosinolate core structure. J. Biol. Chem. 2004, 279, 50717-50725.

18. Smolen, G.; Bender, J. Arabidopsis cytochrome p450 cyp83B1 mutations activate the tryptophan biosynthetic pathway. Genetics 2002, 160, 323-332.

19. Bak, S.; Tax, F.E.; Feldmann, K.A.; Galbraith, D.W.; Feyereisen, R. CYP83B1, a cytochrome $\mathrm{P} 450$ at the metabolic branch point in auxin and indole glucosinolate biosynthesis in Arabidopsis. Plant Cell 2001, 13, 101-111.

20. Naur, P.; Petersen, B.L.; Mikkelsen, M.D.; Bak, S.; Rasmussen, H.; Olsen, C.E.; Halkier, B.A. CYP83A1 and CYP83B1, two nonredundant cytochrome P450 enzymes metabolizing oximes in the biosynthesis of glucosinolates in Arabidopsis. Plant Physiol. 2003, 133, 67-72. 
21. Zang, Y.X.; Kim, H.U.; Kim, J.A.; Lim, M.H.; Jin, M.; Lee, S.C.; Kwon, S.J.; Lee, S.I.; Hong, J.K.; Park, T.H.; et al. Genome-wide identification of glucosinolate synthesis genes in Brassica rapa. FEBS J. 2009, 276, 3559-3574.

22. Bowers, J.E.; Chapman, B.A.; Rong, J.K.; Paterson, A.H. Unravelling angiosperm genome evolution by phylogenetic analysis of chromosomal duplication events. Nature 2003, 422, 433-438.

23. Lysak, M.A.; Koch, M.; Pecinka, A.; Schubert, I. Chromosome triplication found across the tribe Brassiceae. Genome Res. 2005, 15, 516-525.

24. Yang, Y.W.; Lai, K.N.; Tai, P.Y.; Li, W.H. Rates of nucleotide substitution in angiosperm mitochondrial DNA sequences and dates of divergence between Brassica and other angiosperm lineages. J. Mol. Evol. 1999, 48, 597-604.

25. Mizutani, M.; Ward, E.; Ohta, E. Cytochrome p450 superfamily in Arabidopsis thaliana: Isolation of cDNAs, differential expression, and RFLP mapping of multiple cytochromes P450. Plant Mol. Biol. 1998, 37, 39-52.

26. Xu, W.; Bak, S.; Decker, A.; Paquette, S.M.; Feyereisen, R.; Galbraith, D.W. Microarray-based analysis of gene expression in very large gene families: The cytochrome P450 gene superfamily of Arabidopsis thaliana. Gene 2001, 272, 61-74.

27. Brown, P.D.; Tokuhisa, J.G.; Reichelt, M.; Gershenzon, J. Variation of glucosinolate accumulation among different organs and developmental stages of Arabidopsis thaliana. Phytochemistry 2003, 62, 471-481.

28. Gasteiger, E.; Hoogland, C.; Gattiker, A.; Duvaud, S.; Wilkins, M.R.; Appel, R.D.; Bairoch, A. Protein Identification and Analysis Tools on the ExPASy Server. In The Proteomics Protocols Handbook; Walker, J.M., Ed; Humana Press Inc.: Totowa, NJ, USA, 2005; pp. 571-607.

29. Thompson, J.D.; Higgins, D.G.; Gibson, T.J. CLUSTAL W: Improving the sensitivity of progressive multiple sequence alignment through sequence weighting, position-specific gap penalties and weight matrix choice. Nucleic Acids Res. 1994, 22, 4673-4680.

30. Rozen, S.; Skaletsky, H.J. Primer3 on the WWW for General Users and for Biologist Programmers. In Bioinformatics Methods and Protocols: Methods in Molecular Biology; Krawetz, S., Misener, S., Eds.; Humana Press Inc.: Totowa, NJ, USA, 2000; pp. 365-386.

31. Livak, K.J.; Schmittgen, T.D. Analysis of relative gene expression data using real-time quantitative PCR and the $2^{-\Delta \Delta \mathrm{Ct}}$ method. Methods 2001, 25, 402-408.

32. Yin, X.R.; Chen, K.S.; Allan, A.C.; Wu, R.M.; Zhang, B.; Lallu, N.; Ferguson, I.B. Ethylene-induced modulation of genes associated with the ethylene signaling pathway in ripening kiwifruit. J. Exp. Bot. 2008, 59, 2097-2108.

33. Krumbein, A.; Schonhof, I.; Schreiner, M. Composition and contents of phytochemicals (glucosinolates, carotenoids and chlorophylls) and ascorbic acid in selected Brassica species (B. juncea, B. rapa subsp. nipposinica var. chinoleifera, B. rapa subsp. chinensis and $B$. rapa subsp. rapa). J. Appl. Bot. Food Qual. 2005, 79, 168-174.

(C) 2012 by the authors; licensee MDPI, Basel, Switzerland. This article is an open access article distributed under the terms and conditions of the Creative Commons Attribution license (http://creativecommons.org/licenses/by/3.0/). 